\title{
INFLUÊNCIA DO TAMANHO DE PARTÍCULA SOBRE AS PROPRIEDADES TERMOPLÁSTICAS DE CARVÕES COQUEIFICÁVEIS IMPORTADOS E BRASILEIRO*
}

\author{
Matheus Teixeira Fraga ${ }^{1}$ \\ Bruno Deves Flores ${ }^{2}$ \\ Ismael Vemdrame Flores ${ }^{3}$ \\ Luiz G. Schander \\ Guilherme L. R. da Silva ${ }^{5}$ \\ Antônio C. F. Vilela ${ }^{6}$ \\ Eduardo Osório ${ }^{6}$
}

\section{Resumo}

Esse trabalho teve como objetivo avaliar os fenômenos termoplásticos de carvões coqueificáveis e sua relação com o tamanho de partícula. Para isso, dois carvões americanos e um de origem nacional foram amostrados, caracterizados e separados em três tamanhos de partículas. Após a preparação das matérias-primas, ensaios termogravimétricos, de dilatometria e plastometria foram realizados a partir dos diferentes tamanhos de partícula. Os ensaios termoplásticos mostraram que 0 carvão nacional apresentou propriedades plásticas extremamente desenvolvidas e que a granulometria não afeta essas propriedades. No entanto, para os carvões americanos de alto e médio volátil diferenças importantes foram observadas, que indicam uma redução das propriedades termoplásticas com a diminuição do tamanho de partícula.

Palavras-chave: coque; carvão nacional; termoplasticidade; tamanho de partícula.

\section{INFLUENCE OF THE PARTICLE SIZE ON THE THERMOPLASTIC PROPERTIES OF IMPORTED AND BRAZILIAN COKING COALS}

\section{Abstract}

The objective of this paper was evaluating the thermoplastic phenomena of coking coals and its relation with particle size. For this, two American coals and one Brazilian coal were sampled, characterized and separated into three particle sizes. After the raw materials preparation, thermogravimetry, dilatometry and plastometry experiments were carried out on the different particles sizes. The thermoplastic tests showed that the Brazilian coal presents extremely developed plastic properties and particle size does not affect these properties. However, for high and medium volatile American coals significant differences were observed indicating a reduction on thermoplastic properties with a particle size decrease.

Keywords: cokemaking; Brazilian coal; thermoplasticity; particle size.

\footnotetext{
Engenheiro, Laboratório de Siderurgia (LaSid) da UFRGS, Porto Alegre, RS, Brasil.

Mestre, Laboratório de Siderurgia (LaSid) da UFRGS, Porto Alegre, RS, Brasil.

Mestre, Laboratório de processos Metalúrgicos (LAPROMET) da UFMG, Porto Alegre, MG, Brasil. Graduando em engenharia, Laboratório de Siderurgia (LaSid) da UFRGS, Porto Alegre, RS, Brasil.

5 Doutor, Gerdau Ouro Branco, Ouro Branco, MG, Brasil.

6 Professor Dr., Laboratório de Siderurgia (LaSid) da UFRGS, Porto Alegre, RS, Brasil.
} 


\section{INTRODUÇÃO}

O coque metalúrgico é o principal material carbonoso utilizado na redução de óxidos de ferro em altos-fornos, atuando como agente redutor, combustível e garantindo a permeabilidade do processo [1]. O coque é obtido a partir da pirólise de uma mistura de carvões com propriedades adequadas até temperaturas em torno de $1100^{\circ} \mathrm{C}$. No decorrer desse processo, os carvões passam por transformações plásticas no intervalo de temperatura de $350^{\circ} \mathrm{C}$ a $550^{\circ} \mathrm{C}$, durante $\mathrm{o}$ qual a porosidade e a estrutura do coque se desenvolvem [2].

Devido a importante relação entre as propriedades plásticas dos carvões e o processo de coqueificação e qualidade do coque, várias técnicas empíricas foram desenvolvidas para abordar essas propriedades. Testes reológicos para monitorar a plasticidade, inchamento e os poderes aglomerante e coqueificante são comumente utilizados para classificação de carvões, sendo em geral padronizados por normas internacionais [3]. Nesse âmbito, diversos estudos já foram realizados para verificar a influência de parâmetros como granulometria, densidade de carga, pressão, grau de oxidação e taxa de aquecimento sobre a reologia dos carvões [4-10]. Dentre eles, a granulometria do carvão ainda tem sido foco de estudos, uma vez que o tamanho de partícula modifica o desenvolvimento do estágio plástico. Portanto, os processos de britagem de carvões podem ser utilizados como ferramenta para modificar esses parâmetros, em maior ou menor magnitude, de modo a aprimorar a qualidade do coque metalúrgico. Para isso, o conhecimento dos efeitos da granulometria dos carvões sobre suas propriedades plásticas, entre outros, é essencial.

Apesar da pouca literatura específica sobre o assunto, em geral é aceito que a redução do tamanho de partícula diminui as propriedades plásticas dos carvões $[5,11,12]$. Grosh et al. [13] verificou a redução de propriedades plásticas pela granulometria através de ensaios de fluidez e dilatação, respectivamente. Esses autores, semelhante à Loison et al. [5], relacionam a redução de propriedades plásticas com a densidade de carga, uma vez que para uma amostra mais fina e, portanto, de menor densidade, a fase fluida formada escapa facilmente pelo leito mais permeável, participando em menor magnitude nas reações de solubilização.

Nesse sentido, diversos estudos foram realizados com o uso de $\mathrm{H}^{1}$ NMR (Hydrogen1 Nuclear Magnetic Ressonance) com aquecimento in-situ, possibilitando a identificações de fases fluídas e rígidas, bem como a mobilidade das mesmas, durante o desenvolver do estágio plástico, aprofundando o conhecimento sobre o assunto [12,14-16]. De acordo com Maroto-valer et al. [12] a redução da granulometria do carvão causa uma redução significativa na mobilidade da fase plástica, embora sua concentração tenha permanecido constante. Os autores associaram esta redução da mobilidade com a maior área superficial dos inertes obtida na cominuição do carvão, além de possíveis aditivos que possam ser utilizados.

Industrialmente, usualmente carvões são britados de forma a obter distribuições granulométricas entre $70 \%$ e $85 \%$ passante em $3 \mathrm{~mm}[5,17]$. De modo geral, muitos autores consideram que a britagem fina dos carvões aprimora as propriedades do coque metalúrgico, especialmente sua resistência mecânica $[5,18,20]$. Isso ocorre principalmente devido a redução do tamanho dos inertes $[18,5,20]$. Em contrapartida, Lyalyuk et al. [3] diz que para carvões coqueificáveis, cominuição em excesso deve ser evitada, prevenindo a obtenção de partículas menores que $0,5 \mathrm{~mm}$ a um limite máximo de até $32 \%$. Essa proporção garantiria boas propriedades plásticas, bem como densidade de carga satisfatória, garantindo 
uma boa aglomeração entre os inertes pelos reativos e a formação de coque com boa coesão. Ademais, britagem fina deve ser realizada para carvões sem propriedades aglutinantes ou coqueificantes [3].

Embora a influência da granulometria dos carvões pareça bem estabelecida sobre parâmetros individuais como densidade de carga e propriedades plásticas, a compreensão dos conceitos fundamentais responsáveis por cada fenômeno ainda é pouco difundida. Ainda, diferentes abordagens de britagem são realizadas por cada siderúrgica, não sendo observado um consenso de operação ideal. Além disso, a falta de conhecimento junto a grande diversidade de carvões e aditivos e as características operacionais e de mistura de cada empresa, dificultam a otimização dos processos de britagem em busca pela maximização da qualidade do coque. Nesse contexto, esse trabalho avalia a influência da granulometria sobre as propriedades plásticas para um carvão incomum e de fluidez extramente elevada em comparação com carvões usualmente utilizados pela indústria siderúrgica. Para isso foram utilizados testes convencionais de fluidez e dilatrometria, junto a ensaios de termogravimetria e observação em microscópio de aquecimento.

\section{MATERIAIS E MÉTODOS}

\subsection{Carvões}

Para este estudo foram selecionados três carvões. Dois de origem americana, comumente utilizados em misturas para coqueificação, sendo um médio volátil $(\mathrm{Cm})$ e outro alto volátil $(\mathrm{Ca})$. O terceiro carvão é de origem nacional $(\mathrm{CN})$, alto volátil, oriundo de Santa Catarina da região de Criciúma, retirado da camada Barro Branco. A Tabela 1 mostra a caracterização para os carvões de estudo.

Tabela 1. Caracterização dos carvões de estudo

\begin{tabular}{|c|c|c|c|c|c|c|c|c|c|c|}
\hline \multirow[b]{3}{*}{ Unid. } & & & \multicolumn{2}{|c|}{ Análise Imediata } & \multicolumn{2}{|l|}{ Enxofre (S) } & \multicolumn{3}{|c|}{ Análise Petrográfica } & \multirow{3}{*}{ Inertinita } \\
\hline & & & Cinzas & C Fixo & & $\mathrm{Rm}$ & Vitrinita & Liptinita & Semifusinita & \\
\hline & $\%$ bsic & & $\%$ bs & & $\%$ bsic & $\%$ & & & $\%$ imm & \\
\hline $\mathrm{CN}$ & 38,0 & 31,5 & 17,0 & 51,5 & 1,68 & 0,84 & 80,0 & 4,0 & 5,1 & 16,1 \\
\hline $\mathrm{Ca}$ & 36,4 & 34,0 & 6,5 & 59,5 & 1,05 & 0,85 & 69,9 & 12,4 & 9,7 & 17,7 \\
\hline $\mathrm{Cm}$ & 21,8 & 19,8 & 9,0 & 71,2 & 0,92 & 1,29 & 87,3 & 0,0 & 6,5 & 12,7 \\
\hline
\end{tabular}

bsic = base isenta de cinzas; bs = base seca; Vol. = volume; imm = base isenta de matéria mineral; $R m=$ refletância média; $M V=$ matéria volátil; $C$ Fixo = carbono fixo

\subsection{Faixas granulométricas}

Para se avaliar os efeitos de tamanho de partícula sobre as propriedades termoplásticas, os três carvões de estudo foram cominuídos em três distintas faixas granulométricas. As faixas escolhidas foram $<1 \mathrm{~mm},<0,25 \mathrm{~mm}$ e $<0,074 \mathrm{~mm}$. A cominuição das amostras foi realizada manualmente para se evitar ao máximo a formação excessiva de finos. A Figura 1 a-c apresenta a distribuição granulométrica dentro das faixas escolhidas para estudo.
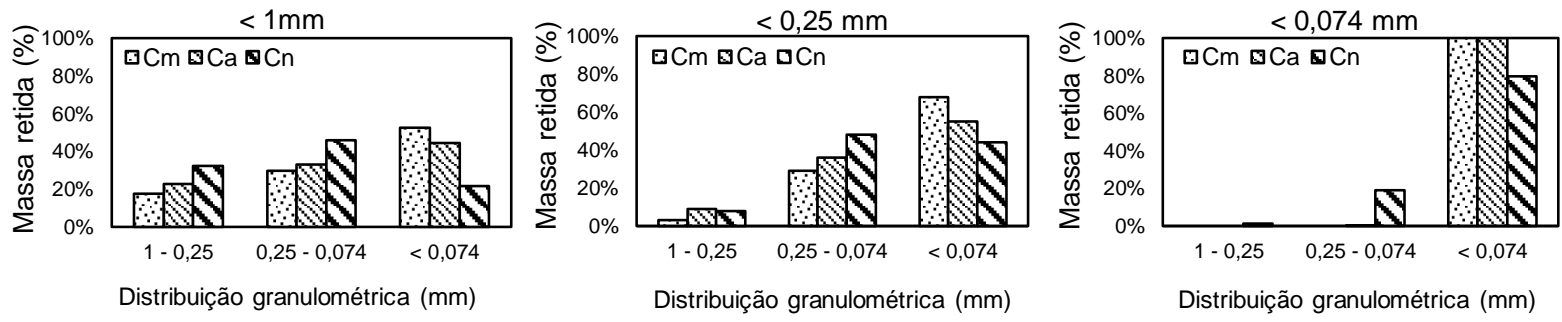

Figura 1. Distribuição granulométrica dentro das faixas escolhidas para o estudo. 


\subsection{Ensaios Termogravimétricos}

As análises termogravimétricas (TGA) dos carvões foram realizadas utilizando uma termobalança da marca NETZSCH, modelo STA 409. Para os ensaios, foram utilizados $50 \mathrm{mg}$ de amostra em cadinho de alumina tipo copo. As amostras de carvão foram aquecidas da temperatura ambiente até $550^{\circ} \mathrm{C}$ (fim do estágio termoplástico) com taxa de $3^{\circ} \mathrm{C} / \mathrm{min}$, mesma taxa dos testes termoplásticos. $\mathrm{O}$ ensaio foi realizado em atmosfera inerte, sob vazão de $50 \mathrm{~mL} / \mathrm{min}$ de $\mathrm{N}_{2}$. A partir da curva de perda de massa foi calculada a taxa de desvolatilização do carvão $(R)$, que é dada pela Equação 1:

$$
\mathrm{R}=-\frac{1}{m o} \times \frac{d m}{d t}
$$

onde m0 é a massa de carvão isenta de cinzas e $\mathrm{dm} / \mathrm{dt}$ a derivada da curva de perda de massa. A partir da curva de desvolatilização dos carvões analisou-se as temperaturas de início de desvolatilização, máxima desvolatilização ( $T_{\text {Rmáx }}$ ), bem como a máxima taxa de desvolatilização ( $\left.R_{\text {máx }}\right)$.

\subsection{Ensaios de dilatometria Audibert-Arnu}

Os ensaios de dilatometria foram realizados a partir de um corpo de prova com o formato cônico, o qual foi produzido com o carvão nas granulometrias especificadas no item 2.2. Inicialmente a amostra é colocada dentro de uma retorta metálica a qual é aquecida entre $300^{\circ} \mathrm{C}$ e $550^{\circ} \mathrm{C}$ a uma taxa de $3^{\circ} \mathrm{C} / \mathrm{min}$. Sobre a amostra de carvão é colocado um pistão cujo deslocamento, devido a variações de altura da amostra, é registrado ao longo do aquecimento. O teste caracteriza o carvão durante o intervalo. Todos os testes foram realizados em duplicata e por utilizar granulometria diferente da norma buscou-se padronizar a densidade de carga de todos os corpos de prova fim de minimizar efeitos das mudanças no leito de partícula. Os parâmetros retirados do ensaio são: temperatura de amolecimento $\left(T_{1}\right)$, temperatura de máxima contração $\left(\mathrm{T}_{2}\right)$, temperatura de ressolidificação $\left(\mathrm{T}_{3}\right)$, máxima contração $(\% \mathrm{C})$ e máxima dilatação (\%D).

\subsection{Ensaios de plastometria Gieseler}

Este teste busca avaliar a fluidez dos carvões, temperatura de amolecimento, ressolidificação e sua máxima fluidez. Essa caracterização é feita pela medida de resistência a rotação de uma haste móvel com torque constante imerso numa massa de carvão de $5 \mathrm{~g}$ submetido a aquecimento pré-definido. Os testes de plastometria foram realizados em duplicatas e as densidades de carga de cada amostra foram registradas para evitar possível efeito devido às diferenças de granulometria. Os parâmetros retirados desse teste são a temperatura de amolecimento $\left(T_{A}\right)$, temperatura de máxima fluidez ( $\left.T_{M F}\right)$, temperatura de ressolidificação ( $\left.T_{R}\right)$ e máxima fluidez (MF). Além disso, a diferença entre temperatura de ressolidificação e temperatura de amolecimento fornece o intervalo plástico.

\subsection{Pirólise de partículas individuais}

A Figura 2 ilustra o aparato experimental utilizado para avaliar o fenômeno termoplástico durante a pirólise de partículas individuais dos três carvões de estudo. $O$ teste foi realizado a partir do aquecimento das amostras em forno resistivo. $O$ 
aquecimento foi feito a partir da temperatura ambiente até $500^{\circ} \mathrm{C}$ a uma taxa de $10^{\circ} \mathrm{C} / \mathrm{min}$, sob fluxo de $100 \mathrm{~mL} / \mathrm{min}$ de $\mathrm{N}_{2}$. Durante aquecimento as partículas foram continuamente filmadas por uma câmera digital. As imagens foram adquiridas aplicando-se aumento digital de $4 x$ para melhor visualização dos fenômenos. A temperatura da partícula de carvão foi monitorada por dois termopares próximos a amostra.

Esses ensaios foram realizados para permitir uma avaliação qualitativa das diferenças termoplásticas dos carvões. Essa avaliação foi feita com partículas de 2 $( \pm 0,5) \mathrm{mm}$, uma vez que granulometrias mais finas dificultam a observação do fenômeno.

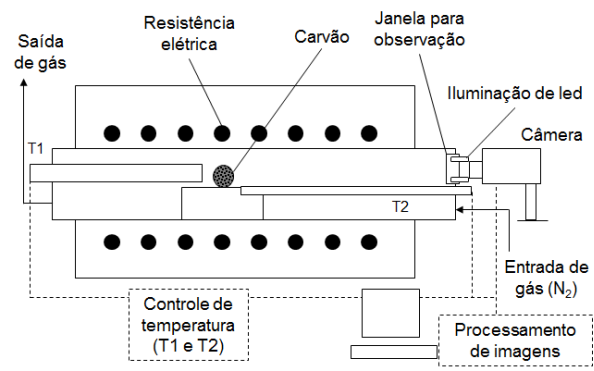

Figura 2. Aparato experimental para avaliação da pirólise de partículas individuais.

\section{RESULTADOS E DISCUSSÃO}

Na Figura 3 encontram-se os gráficos obtidos para os ensaios de termogravimetria (a-c), dilatometria (d-f) e plastometria ( $g$-i) dos três carvões de estudo nas faixas granulométricas $<1 \mathrm{~mm}$, $<0,25 \mathrm{~mm}$ e $<0,074 \mathrm{~mm}$. Nesses gráficos é observada a taxa de perda de massa, contração/dilatação e fluidez das amostras em função da temperatura. Na Tabela 2 é possível observar os parâmetros de interesse obtidos a partir da análise dos gráficos da Figura 3. O item 3.1 irá fazer um comparativo do fenômeno termoplástico entre os carvões somente para o tamanho de partícula de $0,25 \mathrm{~mm}$ (linha sólida na Figura $3 \mathrm{a}$-i). O item 3.2 apresenta a discussão relativa à influência do tamanho de partícula no fenômeno termoplástico desses carvões.

\subsection{Avaliação do fenômeno termoplástico dos carvões}

\subsubsection{Perfil de desvolatilização}

Os testes termogravimétricos (Figura 3 a-c) revelam que em temperaturas próximas a $300^{\circ} \mathrm{C}$, os carvões perdem massa em taxas muito baixas. A perda de massa até essa temperatura correspondeu a menos de $1 \%$ para os três carvões, a qual pode ser atribuída majoritariamente à umidade presente nas amostras. É difícil determinar precisamente em qual momento as reações pirolíticas se iniciam, uma vez que carvões podem perder gases adsorvidos a sua estrutura em temperaturas relativamente elevadas.

Entretanto, nota-se que a partir de $350^{\circ} \mathrm{C}$ a taxa de perda de massa das amostras se intensificam e muito provavelmente as reações pirolíticas tem início. De forma arbitraria, estabeleceu-se a taxa de 0,001 $\mathrm{min}^{-1}$ como início de desvolatilização, permitindo comparar a temperatura na qual cada um dos carvões atinge esse valor ( $\mathrm{T}_{\mathrm{i}}$ - Tabela 2). O carvão nacional $(\mathrm{CN})$ atinge a taxa escolhida na temperatura de $335^{\circ} \mathrm{C}$ (Tabela 2), seguido pelo carvão americano de alto volátil (Ca) $375^{\circ} \mathrm{C}$ e por último o carvão americano de médio volátil $(\mathrm{Cm})$, que só atinge a taxa escolhida na temperatura de $416^{\circ} \mathrm{C}$. É interessante salientar a importante diferença observada 
entre essas temperaturas para os carvões $\mathrm{CN}$ e $\mathrm{Ca}$ (cerca de $40^{\circ} \mathrm{C}$ ), uma vez que esses carvões possuem rank similar (Tabela 1).

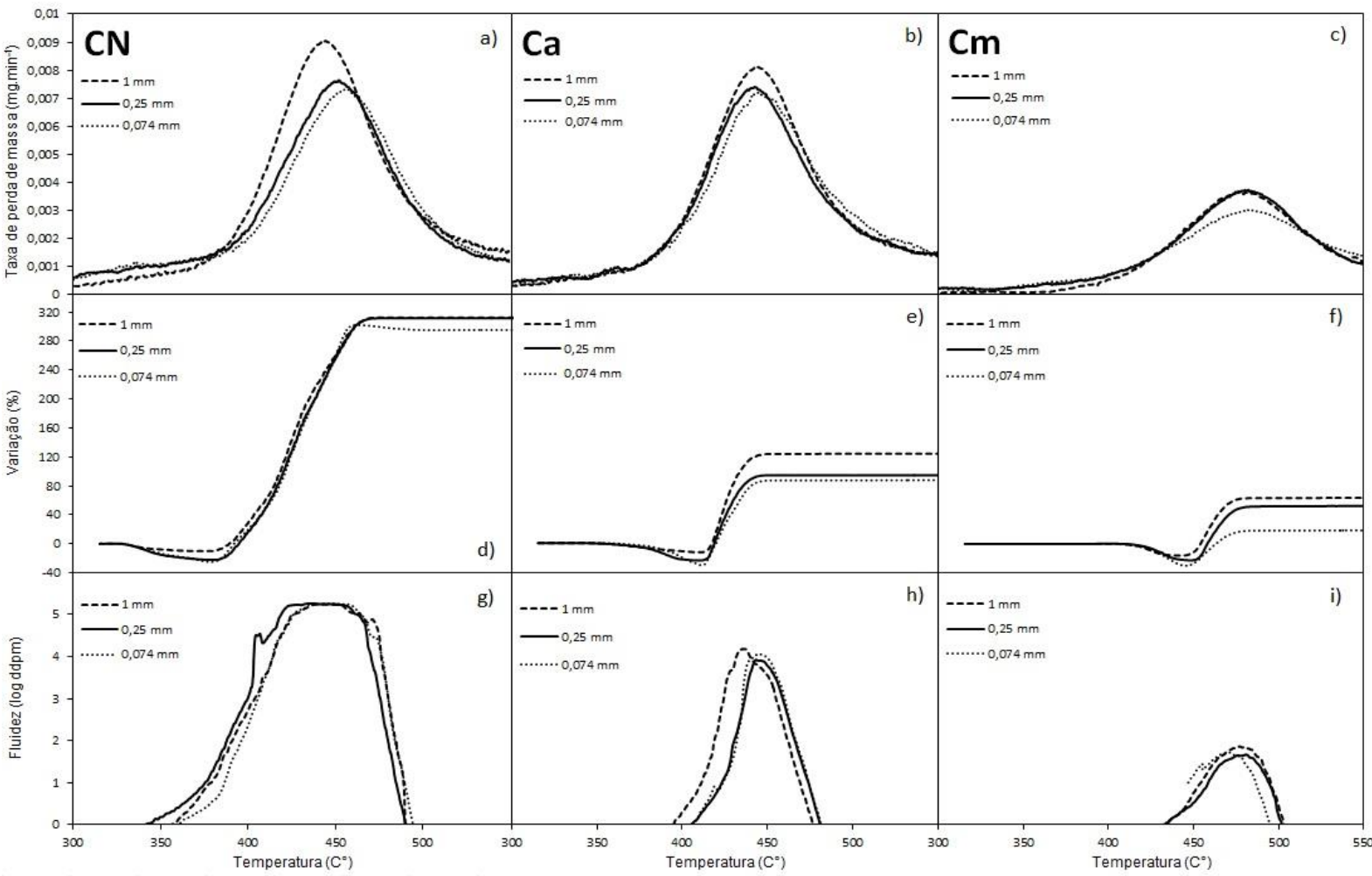

Figura 3. Gráficos obtidos para ensaios de termogravimetria (a-c), dilatometria (d-f) e plastometria ( $g$ i) dos três carvões de estudo nas granulometrias de 1, 0,25 e 0,074mm

Tabela 2. Parâmetros de interesse obtidos a partir da análise dos gráficos

\begin{tabular}{|c|c|c|c|c|c|c|c|c|c|c|c|c|c|}
\hline \multirow[b]{2}{*}{ Carvões } & \multirow{2}{*}{$\begin{array}{c}\text { Tamanho } \\
\text { de } \\
\text { partícula }\end{array}$} & \multicolumn{3}{|c|}{ Termogravimetria } & \multicolumn{5}{|c|}{ Dilatometria } & \multicolumn{4}{|c|}{ Plastometria } \\
\hline & & $\mathrm{T}_{\mathrm{i}}$ & $T_{\text {Rmáx }}$ & $\begin{array}{l}R_{\text {máx }} \\
\min ^{-1}\end{array}$ & $\mathrm{~T}_{1}$ & $\begin{array}{l}T_{2} \\
C^{\circ}\end{array}$ & $\mathrm{T}_{3}$ & $\% \mathrm{C}$ & $\% \mathrm{D}$ & $\mathrm{T}_{\mathrm{A}}$ & $\begin{array}{l}\mathrm{T}_{\mathrm{MF}} \\
\mathrm{C}^{\circ}\end{array}$ & $T_{R}$ & $\begin{array}{c}\text { MF } \\
\text { ddpm }\end{array}$ \\
\hline \multirow{3}{*}{$\mathrm{CN}$} & 1 & 370 & 445 & 0,0091 & 331 & 372 & 472 & -10 & 312 & 355 & 450 & 490 & 173923 \\
\hline & 0,25 & 335 & 452 & 0,0077 & 327 & 377 & 470 & -19 & 314 & 342 & 438 & 490 & 179944 \\
\hline & 0,074 & 330 & 457 & 0,0073 & 331 & 377 & 460 & -25 & 302 & 356 & 448 & 500 & 179917 \\
\hline \multirow{3}{*}{$\mathrm{Ca}$} & 1 & 374 & 444 & 0,0080 & 355 & 409 & 450 & -13 & 123 & 392 & 435 & 486 & 33175 \\
\hline & 0,25 & 374 & 442 & 0,0073 & 352 & 408 & 449 & -24 & 94 & 405 & 443 & 481 & 17409 \\
\hline & 0,074 & 374 & 445 & 0,0071 & 381 & 411 & 453 & -27 & 91 & 405 & 445 & 489 & 24757 \\
\hline \multirow{3}{*}{$\mathrm{Cm}$} & 1 & 419 & 478 & 0,0036 & 413 & 441 & 483 & -16 & 64 & 432 & 477 & 511 & 79 \\
\hline & 0,25 & 416 & 482 & 0,0037 & 420 & 448 & 486 & -23 & 52 & 432 & 481 & 502 & 60 \\
\hline & 0,074 & 414 & 482 & 0,0030 & 415 & 447 & 488 & -29 & 20 & - & 471 & 507 & 73 \\
\hline
\end{tabular}

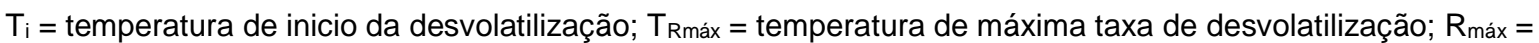
máxima taxa de desvolatilização; $T_{1}=$ temperatura de amolecimento; $T_{2}$ temperatura de contração máxima; $T_{3}=$ temperatura de dilatação máxima; \%C = contração máxima; \%D = dilatação máxima; $\mathrm{T}_{\mathrm{MF}}=$ temperatura de máxima fluidez; $T_{R}=$ temperatura de solidificação; $M F=$ máxima fluidez

O progressivo aumento de temperatura eleva a taxa de desvolatilização das amostras, sendo possível observar um pico acentuado entre $400{ }^{\circ} \mathrm{C}$ e $500^{\circ} \mathrm{C}$, indicando a máxima taxa de perda de massa $\left(R_{\text {máx }}\right)$. Entre os três carvões de estudo, o carvão nacional apresentou a mais alta taxa de desvolatilização $\left(0,0077 \mathrm{~min}^{-1}\right)$, a qual foi atingida em temperatura de $452^{\circ} \mathrm{C}$. 
O carvão Ca apresentou $R_{\text {máx ligeiramente inferior a do carvão nacional }(0,0073 \text { min }}$ ${ }^{1}$ ), a qual foi obtida em temperatura de $442^{\circ} \mathrm{C}$. Já o carvão $\mathrm{Cm}$ apresentou a menor taxa máxima de desvolatilização $\left(0,0037 \mathrm{~min}^{-1}\right)$, a qual foi obtida em temperatura de $482{ }^{\circ} \mathrm{C}$. A partir desses valores, é possível perceber a tendência de diminuição da

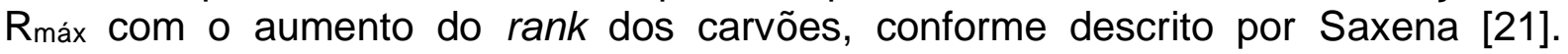
Observa-se que após atingir valor máximo, os carvões sofrem uma rápida redução na taxa de desvolatilização e em temperaturas próximas a $500^{\circ} \mathrm{C}$, as três amostras apresentam taxas similares.

\subsubsection{Perfil de contração/dilatação}

A Figura 3 d-f apresenta os gráficos obtidos no ensaio de dilatometria, onde se observa a variação percentual da altura da amostra em função da temperatura para os três carvões estudados. Valores negativos no gráfico indicam a contração da amostra e valores positivos a sua dilatação. De modo geral, pode-se observar que os carvões apresentam inicialmente contração, a qual é atribuída ao amolecimento das partículas. Após certo período de contração, os carvões iniciam sua dilatação, a qual é atribuída à formação de uma fase "líquida" (metaplasto) e ao processo de desvolatilização, os quais combinados resultam na dilatação da amostra. $O$ fim do processo de dilatação e a ressolidificação dos carvões podem ser identificados pelo patamar estável obtido em temperaturas elevadas.

Entre os três carvões, a amostra $\mathrm{CN}$ apresenta a menor temperatura de amolecimento $\left(327^{\circ} \mathrm{C}\right.$ - Tabela 2) no ensaio de dilatometria (Figura 3 d). Interessante observar que o carvão de rank similar $\mathrm{Ca}$, só inicia amolecimento em temperatura $352^{\circ} \mathrm{C}$ (Figura $3 \mathrm{e}$ ). $\mathrm{O}$ carvão $\mathrm{Cm}$ apresenta a maior temperatura de amolecimento entre as amostras $\left(420^{\circ} \mathrm{C}\right.$ - Figura $\left.1 \mathrm{f}\right)$. O fenômeno de dilatação do carvão nacional $(\mathrm{CN})$ se inicia em temperatura próxima a $375^{\circ} \mathrm{C}$ e continua até $470{ }^{\circ} \mathrm{C}$. A máxima dilatação da amostra $\mathrm{CN}$ foi de $314 \%$, valor extremamente elevado e considerado atípico para carvões betuminos [5]. O carvão americano $\mathrm{Ca}$, inicia sua dilatação em temperatura de $408^{\circ} \mathrm{C}$ e apresenta seu valor máximo de dilatação $(94 \%)$, em temperatura de $449^{\circ} \mathrm{C}$. Já o carvão de médio volátil $\mathrm{Cm}$ apresenta a menor dilatação entre as amostras e em temperaturas mais elevadas que os demais carvões.

\subsubsection{Perfil de plasticidade}

A Figura $3 \mathrm{~g}$-i apresenta as curvas obtidas no ensaio de plastometria dos carvões $\mathrm{CN}$, $\mathrm{Ca}$ e $\mathrm{Cm}$, respectivamente. De modo geral, observa-se que a fluidez dos carvões aumenta com o progressivo aumento da temperatura até atingir um máximo. É nesse intervalo que ocorre a máxima formação da fase plástica dos carvões, responsável pelo comportamento de fluidez das amostras. A fluidez dos carvões diminui devido ao processo de ressolidificação da amostra. $O$ intervalo de temperatura entre o amolecimento da amostra e sua ressolidificação é conhecido com intervalo plástico.

Observa-se que o carvão nacional inicia seu amolecimento em temperatura de $341^{\circ} \mathrm{C}$ (Tabela 2). Após o início de amolecimento, carvão nacional apresenta um rápido aumento de fluidez, atingindo valor acima da capacidade de detecção do equipamento utilizado (aproximadamente $180000 \mathrm{ddpm}$ ). Isso pode ser identificado pelo aparente patamar de fluidez observado entre as temperaturas de $410{ }^{\circ} \mathrm{C} \mathrm{e}$ $460{ }^{\circ} \mathrm{C}$. Assim, não foi possível identificar a precisa temperatura de máxima fluidez para o carvão nacional. Logo, os valores de máxima fluidez (MF) e temperatura de 
máxima fluidez ( $\mathrm{T}_{\mathrm{MF}}$ ), observados na Tabela 2, possuem certa imprecisão. O carvão nacional apresenta ressolidificação em temperatura de $490^{\circ} \mathrm{C}$. É importante destacar que o comportamento de fluidez do carvão nacional é atípico para carvões betuminosos, tanto pelos altos valores de fluidez, bem como pelo largo intervalo plástico. Nota-se que o carvão $\mathrm{Ca}$, apresenta temperatura de amolecimento em $405^{\circ} \mathrm{C}$ e atinge sua máxima fluidez (17409 ddpm) em $443^{\circ} \mathrm{C}$. Comparativamente, o carvão de médio volátil $\mathrm{Cm}$ possui o seu intervalo plástico em temperaturas mais elevadas $\left(\mathrm{T}_{\mathrm{a}}=432{ }^{\circ} \mathrm{C} \mathrm{T}_{\text {res }}=501^{\circ} \mathrm{C}\right)$ e a menor fluidez entre as amostras (60 ddpm). O comportamento de fluidez de carvões betuminosos está fortemente associado ao seu grau de carbonificação, onde carvões de mais baixo rank apresentam mais alta fluidez [5], fato que justifica a diferença observada entre os carvões importados.

\subsubsection{Correlação entre os testes termoplásticos}

A desvolatilização (Figura 3 a-c) dos carvões está nitidamente associada aos comportamentos de contração/dilatação (Figura 3 d-f) e fluidez (Figura $3 \mathrm{~g}-\mathrm{i}$ ) observados. As temperaturas de início de desvolatilização e amolecimento das amostras são relativamente similares (Tabela 2). Aparentemente, as reações pirolíticas se iniciam em temperaturas ligeiramente inferiores ao amolecimento ser detectado, seja no teste de dilatometria ou plastometria. A temperatura na qual os carvões apresentam sua máxima taxa de desvolatilização ( $T_{\text {Rmáx }}$ ) é bastante similar a temperatura de máxima fluidez $\left(T_{M F}\right)$. No entanto, curiosamente os carvões atingem a máxima dilatação em temperaturas inferiores a TRmáx.

Para ilustrar o desenvolvimento termoplástico dos carvões de estudo, testes de pirólise com partículas individuais foram realizados. Esses testes permitem a visualização do fenômeno e auxiliam correlacionar as transformações descritas nos testes de caracterização. A Figura 4 mostra as transformações das partículas dos diferentes carvões durante o ensaio de pirólise. Da esquerda para direita são apresentadas as imagens das partículas para as temperaturas de relevância durante os ensaios de termogravimetria ( $T_{i}$ e $\left.T_{R m a ́ x}\right)$ dilatometria $\left(T_{3}\right)$ e plastometria $\left(T_{A}, T_{M F}\right.$, $T_{R}$ ), conforme Tabela 2. As imagens foram alinhadas de forma cronológica para se observar a ocorrência dos fenômenos com o progressivo aumento de temperatura.

Observa-se que para as três amostras não são perceptíveis transformações entre as temperaturas de início de desvolatilização $\left(\mathrm{T}_{\mathrm{i}}\right)$ e amolecimento ( $\left.\mathrm{T}_{\mathrm{A}}\right)$. No entanto, é notável o inchamento das partículas ao atingirem a temperatura de máxima fluidez. Para o carvão nacional observa-se que o inchamento ocorreu até a temperatura de máxima dilatação ( $\left.\mathrm{T}_{3}\right)$. Já para os carvões $\mathrm{Ca}$ e $\mathrm{Cm}$ não foram observadas alterações significativas após as amostras atingirem a temperatura de máxima fluidez.

Os ensaios de pirólise mostram claramente que o carvão nacional apresenta propriedades termoplásticas extremamente desenvolvidas. Nas condições do teste, o excesso de formação de fase plástica aliada a desvolatilização das partículas de CN resulta na formação de bolhas, fazendo com que a massa carbonosa tenha uma expansão muito superior a dos demais carvões.

\subsubsection{Influência do tamanho de partícula no fenômeno termoplástico}

A avaliação do efeito do tamanho de partícula sobre as propriedades termoplásticas dos carvões foi realizada a partir de três distintas distribuições granulométricas $(<1$ $\mathrm{mm},<0,25 \mathrm{~mm} \mathrm{e}<0,074 \mathrm{~mm}$ ), conforme discutido no item 2. Na Figura 2 a-i a linha 
pontilhada representa as amostras com distribuição $<1 \mathrm{~mm}$, a linha sólida representa as partículas $<0,25 \mathrm{~mm}$ (discutido no item 3.1) e a linha pontilhada as amostras com granulometria inferior a $0,0074 \mathrm{~mm}$.

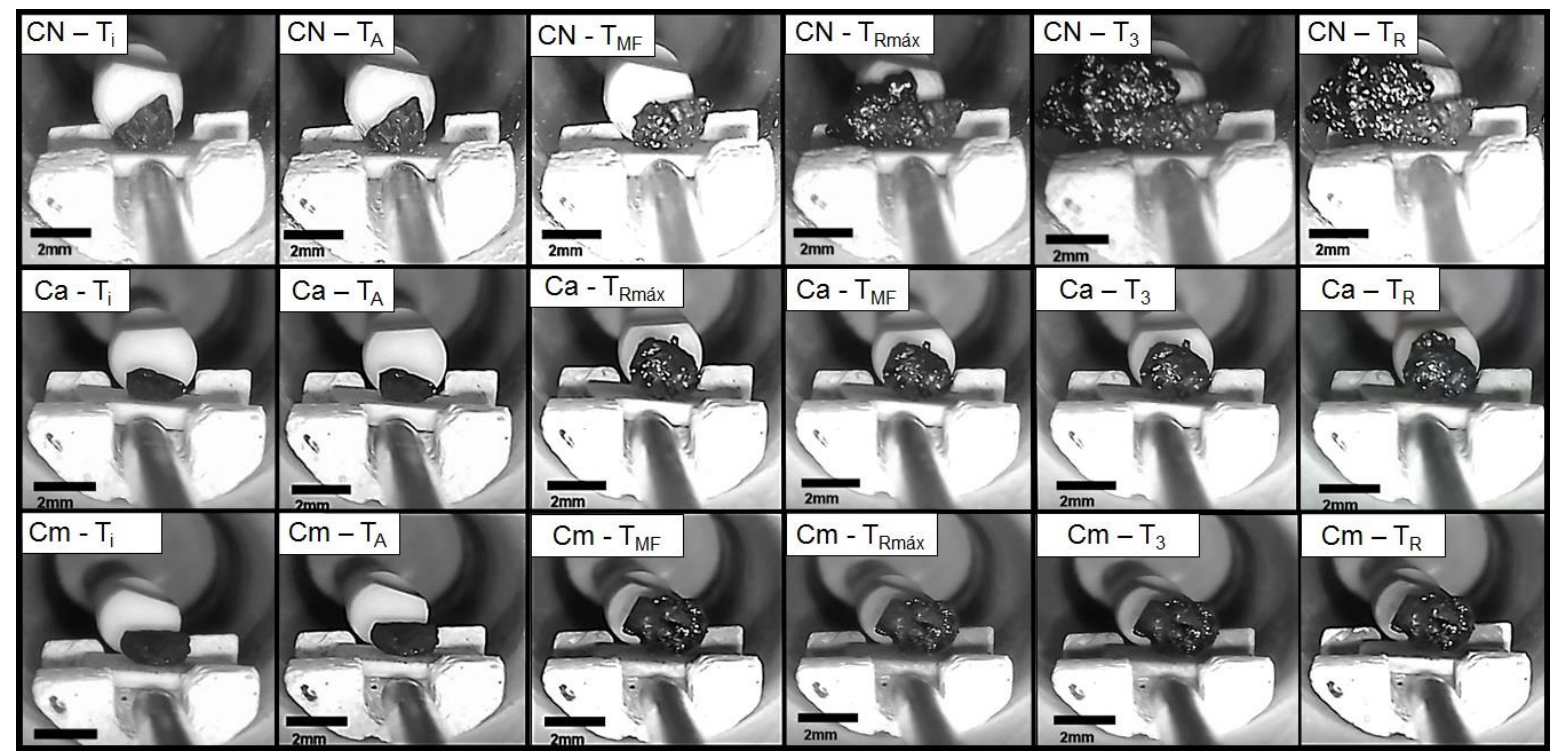

Figura 4. Avaliação in situ das transformações termoplásticas em carvões coqueificáveis.

\subsubsection{Efeito sobre a taxa de desvolatiliazação dos carvões}

A partir dos testes de termogravimetria (Figura $3 \mathrm{a}-\mathrm{c}$ ), observa-se que a temperatura de início de desvolatilização (Tabela 2) diminui com a redução do tamanho de partícula para os carvões $\mathrm{CN}$ e $\mathrm{Cm}$. Essa redução foi mais importante para o carvão $\mathrm{CN}$ ao se reduzir sua faixa granulométrica de $<1$ para $<0,25 \mathrm{~mm}$. A máxima taxa de desvolatilização foi claramente afetada pelo tamanho de partícula dos carvões. Para os dois carvões com propriedades termoplásticas mais desenvolvidas ( $C N$ e $\mathrm{Ca}$ ), observa-se um aumento da taxa de desvolatilização para partículas mais grosseiras. A temperatura onde a taxa de desvolatilização apresenta seu máximo valor foi pouco afetada pelo tamanho de partícula dos carvões, com uma leve redução de $T_{\text {Rmáx }}$ com o aumento do tamanho de partícula do carvão CN. Dentre os carvões estudados, $\mathrm{Cm}$ apresenta as menores variações do perfil de desvolatilização para diferentes tamanhos de partícula.

É sabido que o tamanho de partícula dos carvões durante desvolatilização afeta os mecanismos de transferência de massa e calor no interior das partículas e que esses mecanismos tem influência no tempo de desvolatilização. O esperado é que à medida que o tamanho de partícula aumente, a resposta térmica da partícula diminua. Isto significa que para um dado nível de desvolatilização partículas maiores levarão mais tempo [21]. A tendência geral observada no estudo foi contrária a essa descrição. No entanto, Saxena [21] destaca que os mecanismo de desvolatilização são afetados fortemente pelas transformações termpoplásticas dos carvões, uma vez que após o amolecimento as partículas aglomeram e não se comportam como partículas individuais. É possivel que em particulas mais grosseira a aglutinação do leito tenha sido menos pronunciada, permitindo uma desvolatilização mais rápida da massa carbonosa. No entanto, estudos futuros deverão ser realizados para esclarecer essa hipótese. 


\subsubsection{Efeito sobre a contração/dilatação dos carvões}

O ensaio de dilatometria para os diferentes tamanhos de partícula (Figura $3 \mathrm{~d}$-f) revelam que quanto menor o tamanho de partícula maior a contração dos carvões. Já na dilatação o efeito do tamanho de partícula pode ser claramente observado para as amostra $\mathrm{Ca}$ e $\mathrm{Cm}$, nas quais a redução granulométrica leva a redução da dilatação dos carvões. Para o carvão nacional observa-se que as variações granulométricas não resultam em diferenças apreciáveis na dilatação máxima das amostras. Não se observou correlações entre as temperaturas características do teste e a variação granulométrica dos carvões.

\subsubsection{Efeito sobre plastometria dos carvões}

Os testes de plastometria apresentados na Figura $3 \mathrm{~g}$-i mostram que as variações de tamanho de partícula apresentam influência na fluidez dos carvões $\mathrm{Ca}$ e $\mathrm{Cm}$. Os resultados indicam que a fluidez desses carvões é mais elevada para a faixa granulométrica mais grosseira. A máxima fluidez das amostras é reduzida nas amostras com tamanho $<0,25 \mathrm{~mm}$. No entanto, se observa um relativo aumento da máxima fluidez ao se diminuir o tamanho das partículas a $<0,074 \mathrm{~mm}$. Acredita-se que esses resultados podem ser explicados por dois fatores concorrentes. $O$ primeiro é a redução intrínseca da formação de material plástico devido à redução do tamanho de partícula. Yip [22] ressalta o fato de que partículas maiores dificultam a evolução dos gases, provavelmente aumentando a pressão interna e assim condensando parte dos gases metaestáveis, o que acarretaria em um aumento de material na fase plástica. O segundo se deve a variação da densidade de carga das amostras devido à variação granulométrica. A Figura 4 mostra a variação da densidade de carga das amostras carregadas no plastômetro em função do tamanho de partícula, onde se observa uma clara redução da densidade para partículas de menor tamanho. Assim, acredita-se que o aumento da fluidez para partículas de tamanho $<0,074 \mathrm{~mm}$ seja resultado da menor densidade do leito de partículas, fato que aumentando a facilidade de a haste girar e influenciando na medição do aparelho. Não se observou correlações entre as temperaturas características do teste e a variação granulométrica desses carvões.

Para o carvão nacional (CN) não houve influência do tamanho de partícula sobre os valores de máxima fluidez (MF). No entanto, é importante destacar que os valores de fluidez máxima para as três granulometrias testadas estão acima do limite de detecção do instrumento utilizado. Portanto, é possível apenas afirmar que nas condições testadas essas granulometrias não apresentam diferenças.

\subsubsection{Relevância dos resultados para a utilização de carvão nacional na fabricação de coque}

A partir dos testes de caracterização descritos acima, é possível avaliar as transformações que carvões coqueificáveis estão submetidos durante aquecimento. Conforme mencionado, o carvão nacional apresenta propriedades termoplásticas atípicas para carvões betuminosos. Isso fica evidente a partir dos testes de dilatometria e plastometria, nos quais se evidenciou sua alta dilatação e fluidez, bem como seu largo intervalo plástico. Chama a atenção, que carvões de rank similar e com perfil de desvolatilização semelhantes como $\mathrm{CN}$ e $\mathrm{Ca}$ possam apresentar tamanha diferença no seu comportamento termoplástico. Não é de conhecimento dos autores, que esse comportamento tenha sido até o presente momento explicado em detalhes. 
Do ponto de vista da coqueificação, essas propriedades podem trazer benefícios, uma vez que carvões com elevadas propriedades termoplásticas podem auxiliar na aglutinação de partículas inertes durante a carbonização. De forma oposta, o excesso de plasticidade pode causar porosidade irregular e excessiva, levando a formação de um coque de baixa resistência.

Verificou-se no estudo que as propriedades termoplásticas do carvão nacional não são alteradas pelo tamanho de partícula do material. Assim, quando inserido em misturas, o carvão nacional pode ser adicionado em granulometrias finas, a fim de maximizar sua dispersão na mistura e consequentemente aumentar seu poder aglutinante. $O$ ajuste granulométrico do carvão nacional pode auxiliar também na formação de uma matriz microporosa mais adequada, evitando a formação de grandes poros de formato irregular, conforme ilustrado na Figura 5. A soma de ambos os fatores devem produzir coques de maior resistência.

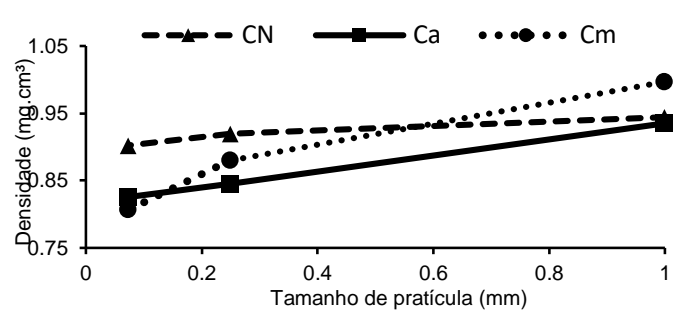

Figura 4. Média das densidades medidas para cada amostra.

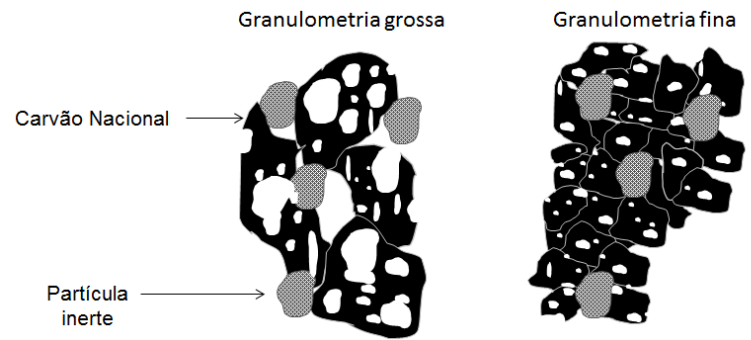

Figura 5. Formação da estrutura porosa em função do tamanho de partícula do carvão nacional

\section{CONCLUSÃO}

O presente trabalho buscou avaliar os fenômenos termoplásticos de carvões coqueificáveis e sua relação com o tamanho de partícula. Os ensaios termoplásticos mostram claramente que o carvão nacional apresenta propriedades plásticas extremamente desenvolvidas. Para esse carvão a variação granulométrica não afetou de forma relevante as propriedades termoplásticas nas condições testadas. Do ponto de vista da coqueificação, essas propriedades podem trazer benefícios, uma vez que granulometrias finas maximizam sua dispersão na mistura auxiliando na aglutinação de partículas inertes durante a carbonização. No entanto, para os carvões americanos de alto e médio volátil diferenças importantes foram observadas, que indicam uma redução das propriedades termoplásticas com a diminuição do tamanho de partícula.

\section{Agradecimentos}

Os autores desse trabalho agradecem a Gerdau Usina Ouro Branco pelo fornecimento dos carvões importados e ao Conselho Nacional de Desenvolvimento Científico e Tecnológico (CNPq) pelo incentivo financeiro.

\section{REFERÊNCIAS}

1. Díez, MA, Alvarez R, Barriocanal C. Coal for metallurgical coke production: predictions of coke quality and future requeriments ofr cokemaking. International Journal of Coal Geology, v. 50, p. 389-412, 2002. 
2. Gray RJ. Theory of carbonization of coal. The first international meeting on coal and coke applied to coke making. Brazillian Society of Metals, ABM, August 9-15, p. 551579, 1987.

3. Lyalyuk VP, Uchitel AD, Lyakhova IA, Kassim DA, Zaitsev GL. Preparation of coking batch. Coke and Chemistry, v. 54, n. 8, pp. 271-286, May 2011.

4. Maroto-Valer MM, Atkinson CJ, Willmers RR, Snape CE. Characterization of partially carbonized coals by solid-state C NMR and optical microscopy. Energy and Fuels. v. 12, n. 5, p. 833-842, 1998.

5. Loison R, Foch P, Boyer A. Coke: Quality and Production. 2nd ed. England: Butterworths, 1989.

6. Nomura S, Arima T, Kato K. Coal blending theory for dry coal charging process. Fuel, v. 83, pp. 1771-1776, 2004.

7. Standish N, Yu AB, Zou RP. Optimization of coal grind for maximum bulk density. Powder Technology, Wollongong, v. 68, pp.175-186, May 1991.

8. Blayden HE, Noble W, Rilley HL. The influence of carbonizing conditions on coke properties. Part I. Mechanical Pressure. Jour. Iron and Steel Inst. v. 136, p. 47-76, 1937.

9. Cimadevilla JLG, Álvarez R, Pis JJ. Effect of coal weathering on thechnological properties of cokes produced at different scales. Fuel Processing Technology, v. 86, p. 809-830, 2005.

10. Casal MD, González AI, Canga CS, Barriocanal C, Pis JJ, Alvarez R, Díez MA. Modifications of coking coal and metallurgical coke propeties induced by coal weathering. Fuel Processing Technology, v. 84, p. 47-62, 2003.

11. Ryan B. Fluidity of western Canadian coals and its relationship to other coal and coke properties. Geological Fieldwork, British Columbia Geological Survey, 1997.

12. Maroto-Valer MM, Andrésen JM, Snape CE. In-situ H1 NMR Investigation of particle size, mild oxidation and heating regime effects on plasticity development during coal carbonization. Energy \& Fuels, v. 11, p. 236-244, 1997.

13. Ghosh SR, Das Gupta NN, Lahiri AJJ. Sci. Ind. Res. v. 16, n. 89, 1957.

14. Maroto-Valer MM, Andrésen JM, Snape CE. In situ H NMR study of the fluidity enchancement for bituminous coal by coal tar pitch and a hydrogen-donor liquefaction residue. Fuel, v. 77, n. 9/10, p. 921-926, 1998.

15. Maroto-Valer MM, Andrésen JM, Snape CE. Quantification by in situ H n.m.r. of the contributions from pyridine-extractables and metaplast to the generation of coal plasticity. Fuel, v. 76, n. 13, p. 1301-1308, 1997

16. Sakurovs R. Interactions between coking coals in blends. Fuel, v. 82, p. 439-450, 2003.

17. O'Brian G, Warren K, Mahoney M, Pickup A. Quantifying the influence that the composition of the individual coal grains in coke oven feed has on coke quality. Acarp Project C16047. 2010.

18. Kubota $\mathrm{Y}$, Nomura S, Arima $\mathrm{T}$, Kato K. Effects of coal inertinite size on coke strength. ISIJ International, v. 48, n. 5, p. 563-571, 2008.

19. Miroshnichenko DV. Influence of coal preparation and coking conditions on coke reactivity. Coke and Chemistry, v. 52, n. 2, p. 77-82, 2009.

20. Kaye JCA, Horton AE. Composition and preparation of blends for making metallurgical coke. Commission of the European communities, Coke oven techniques, information symposium. Luxembourg, 6 e 7, Maio, 1975.

21. Saxena SC. Devolatilization and combustion characteristics of coal particles. Prog. Energy Combust. Sci. 1990, Vol. 16, pp. 55-94.

22. Yip K, Wu H, Zhang DK. Effects of inherent moisture is collie coal on its pyrolysis due to in-situ steam gasification, Energy and Fuels, v. 21, pp. 2883-2891, 2007. 